\title{
OFDM-IM Based Dual-Hop System Using Fixed-Gain Amplify-and-Forward Relay with Pre-Processing Capability
}

\author{
Shuping Dang, Member, IEEE, Jun Li, Member, IEEE, Miaowen Wen, Senior Member, IEEE, \\ Shahid Mumtaz, Senior Member, IEEE, and Zhenrong Zhang, Member, IEEE
}

\begin{abstract}
Orthogonal frequency-division multiplexing with index modulation (OFDM-IM) has recently attracted much researchers' attention due to its superior spectrum efficiency and reliability compared to the traditional OFDM. Cooperative decode-and-forward (DF) relaying has been incorporated with OFDM-IM, which provides a higher energy efficiency and better network coverage. However, it might not be feasible in realistic applications owing to the high system complexity and transmission delay rendered by complex decoding and channel estimation procedures. Therefore, in this paper, we propose a fixed-gain (FG) amplify-and-forward (AF) relay assisted OFDMIM system, which does not need to perform complex decoding and channel estimation at the relay, but only requires a preprocessing capability at the relay, e.g., cyclic prefix (CP) removal and re-insertion. Therefore, the system complexity can be reduced and the forwarding delay as well as power consumption caused by processing at the relay also decline. We analyze the average outage probability, block error rate (BLER), and achievable rate of the proposed system and verify all analysis by numerical results. The proposed FG AF relay assisted OFDM-IM provides a simple solution to the implementation of OFDM-IM in new network paradigms, where nodes are simple, power-limited and/or complexity-limited.
\end{abstract}

Index Terms-Orthogonal frequency-division multiplexing with index modulation (OFDM-IM), fixed-gain (FG) amplifyand-forward (AF) relaying, performance analysis, multi-carrier systems, the Internet of Things (IoT).

\section{INTRODUCTION}

This work was supported in part by the National Natural Science Foundation of China under Grant 61871190 and Grant 61701127 , in part by the Natural Science Foundation of Guangdong Province under Grant 2018B030306005, in part by the Pearl River Nova Program of Guangzhou under Grant 201806010171, and in part by the Young Elite Scientists Sponsorship Program by CAST under Grant 2018QNRC001. (Corresponding author: Miaowen Wen.)

S. Dang was with the R\&D Center, Guangxi Huanan Communication Co., Ltd., Nanning 530007, China when submitting the first version of this paper, and is now with Computer, Electrical and Mathematical Science and Engineering Division, King Abdullah University of Science and Technology (KAUST), Thuwal 23955-6900, Kingdom of Saudi Arabia (e-mail: shuping.dang@kaust.edu.sa).

J. $\mathrm{Li}$ is with the School of Mechanical and Electrical Engineering, Guangzhou University, Guangzhou 510006, China (e-mail: lijun52018@gzhu.edu.cn).

M. Wen is with the School of Electronic and Information Engineering, South China University of Technology, Guangzhou 510641, China (e-mail: eemwwen@scut.edu.cn).

S. Mumtaz is with Instituto de Telecomunicações, Campus Universitário de Santiago, Aveiro 3810-193, Portugal (e-mail: smumtaz@av.it.pt)

Z.R. Zhang is with the School of Computer, Electronics and Information, Guangxi University, and Guangxi Key Laboratory of Multimedia Communications and Network Technology, Nanning 530004, China (e-mail: zzr76@gxu.edu.cn).
$\mathbf{O}$ RTHOGONAL frequency-division multiplexing (OFDM) has been adopted as one of the core modulation technologies in fourth generation (4G) wireless networks and relevant standardization activities have been completed. These advantages make OFDM a mature and promising candidate for next generation wireless networks [1]. On the other hand, recent developments of wireless networks propose new challenges and service requirements for modulation technologies. Specifically, much higher data rate and reliability should be supported by rapidly increasing numbers of wireless service subscribers and machine-type communication (MTC) nodes [2]. To cope with these new challenges and the heterogeneous service requirements, various novel modulation techniques are invented. Two of the most well-known modulation techniques are the spatial modulation (SM) and OFDM with index modulation (OFDMIM) [3], both involving an extra modulation dimension apart from the conventional signal amplitude and phase dimensions. The former resorts to the index of antenna activation pattern (AAP) in multiple-input multiple-output (MIMO) systems [4], while the latter utilizes the index of subcarrier activation pattern (SAP) in OFDM systems [5].

OFDM-IM, taking the advantage of the mature standardization, existing infrastructure and achievements of OFDM, has been regarded as a propitious candidate modulation scheme for fifth generation (5G) wireless networks [6]. OFDM-IM has also been proven to outperform the traditional OFDM regarding reliability and throughput under certain conditions [3], [7]. More importantly, the deployment of OFDM-IM hardly requires modification of hardware equipment, but only updates the modulation/demodulation process, which can be easily realized in the software level [8]. The achievable rate of OFDM-IM is examined in [9]. Several enhanced derivatives of the conventional OFDM-IM are proposed in [10]-[12], which are suited for different application scenarios. Coordinate interleaving is employed in OFDM-IM systems to achieve a diversity gain in [13]. Multi-mode OFDM-IM has been systematically investigated in [14]. Besides, subcarrier allocation is adopted as a key technique to enhance the error performance of OFDM-IM in [15] and [16]. Additionally, a comprehensive literature survey of OFDM-IM is provided in [17].

\section{A. Related Works of Relay Assisted OFDM-IM}

On the contrary, OFDM-IM has a relatively low energy efficiency compared to its spatial counterpart-SM, owing to 
the lack of beamforming capability. In order to enhance the energy efficiency and provide a larger coverage for OFDMIM systems, relay technology has been involved to form the paradigm of relay assisted OFDM-IM. This cooperative paradigm can well offset the inherent disadvantages of OFDMIM. In [18], a primary numerical study of relay assisted OFDM-IM is first presented. In [19], relay assisted OFDMIM is applied in cognitive radio (CR) networks. Following these two works, relay assisted OFDM-IM is further enhanced by subcarrier permutation, power allocation, and various relay selection in [20]-[23], respectively. Besides, multi-hop and full-duplex relay assisted OFDM-IM systems are investigated in [24] and [25].

\section{B. DF and AF Relaying: Pros and Cons}

Although insightful, all aforementioned works only dedicate to the decode-and-forward (DF) relaying protocol owing to its analytical simplicity and mathematical tractability. DF relaying belongs to the category of non-transparent relaying techniques that process the received information by decoding and reencoding. DF relaying has the following features [26]:

- Full instantaneous channel state information (CSI) is required.

- More complicated processing modules and units, and thereby a higher computational complexity are required.

- More energy is consumed by performing sophisticated processing.

- A higher end-to-end transmission delay is expected.

- More difficult to handle the channel imbalance over multiple hops owing to error propagation.

Because of these features, DF relaying is more suited for the application scenarios, in which 1) intermediate nodes are equipped with powerful processing capability and reliable power supply; 2) the network is delay-tolerant; 3) fading environments over different hops are similar and less volatile (slow fading). If the above three conditions are not satisfied, DF relaying would bring new challenges that could even render worse performance. In particular, frequent channel estimation for obtaining instantaneous CSI will result in a huge amount of extra signaling overhead and soon drain the energy of a relay node. A higher end-to-end delay and/or outdated signaling information could lead to severe network congestion and even system-level breakdown in some delay-sensitive scenarios, e.g., vehicle-to-everything (V2X) communication networks and a variety of special MTC networks for control and security [27]-[29]. However, the powerful processing capability and reliable power supply might be hardly equipped in some new network paradigms, where intermediate nodes are with simple structures and powered by non-rechargeable batteries, e.g., wireless sensor networks (WSNs) and some application scenarios of the Internet of Things (IoT) [30], [31].

To deal with these practical implementation issues, another relaying scheme-amplify-and-forward (AF) relaying classified into the category of transparent relaying techniques attracts researchers' attention. AF relaying is the simplistic relaying technique, and an AF relay is, in essence, an analog repeater performing power scaling. The concept of fixed-gain
(FG) AF relaying is proposed in [32] in order to get rid of the requirement of instantaneous CSI. Then, its performance analysis has been generalized to a multi-hop architecture and an arbitrary fading environment in [33] and [34], respectively. The asymptotic performance and diversity order of FG AF relay systems are studied in [35]-[37]. Some practical implementation issues and application scenarios vis-à-vis FG AF relaying are investigated in [38]. More closely related to the study in this paper, FG AF relaying is incorporated with $M$-ary phase shift keying ( $M$-PSK) and SM systems in [39] and [40], respectively. Opposite FG AF relaying that only requires the statistical CSI for the first hop (this reduces the CSI requirement to the minimum), another well-known $\mathrm{AF}$ relaying scheme is termed the (VG) AF relaying, which requires the instantaneous CSI to set up the amplification gain in a real-time manner [41]. A detailed comparative study between these two AF relaying schemes is presented in [36].

Admittedly, it is impossible to identify one relaying scheme capable of outperforming others in all cases, and different relaying schemes have their own pros and cons as well as application scenarios [42]. To fully exploit the benefits of cooperative relaying and mitigate the rendered drawbacks, one should always deliberate and take practical conditions and required performance into account when selecting relaying scheme. That is, one should also note that DF relaying might not always outperform AF relaying due to the error propagation problem, and it has been rigorously proven that there exists a performance cross point between AF and DF relaying [26]. This is because it is likely that the received signal at the DF relay would be erroneously decoded, and the re-encoded signal will be different from the originally transmitted signal at the source. This also refers to the wellknown bottleneck rendered by DF relaying.

\section{Contributions and Organization}

Considering the overall system requirements in new network paradigms, we choose FG AF relaying to assist the transmission of OFDM-IM systems in order to minimize the CSI requirement, and propose an FG AF relay assisted OFDMIM scheme in this paper. By applying the FG AF relaying protocol, the relay node neither estimates wireless channels nor decodes received signals, but only needs to perform some preprocessing operations prior to amplifying and re-transmitting received signals. Rather, it simply amplifies the received signal by a fixed amplification gain and forwards the amplified signal to the destination. In this way, we can achieve a lowcomplexity and fast relay assisted OFDM-IM system, and save the processing power for the relay node in the meantime.

To provide insight into the proposed FG AF relay assisted OFDM-IM system in a comprehensive manner, we analyze the outage performance, error performance and achievable rate of a generic FG AF relaying scheme with an arbitrary amplification gain, and then concrete the generic analysis into several special examples with different amplification gain configurations. Specifically, the average outage probability, block error rate (BLER) and achievable rate of the conventional modulation symbols are derived or approximated in closed 
TABLE I: Functions and notations used in this paper

\begin{tabular}{|c|c|}
\hline Notation & Definition/explanation \\
\hline $\mathbb{E}\{\cdot\}$ & $\begin{array}{l}\text { Expected value of the enclosed random } \\
\text { variable }\end{array}$ \\
\hline $\operatorname{Ei}(z)$ & Exponential integral function \\
\hline $\exp (z)$ & Exponential function \\
\hline$G_{n, n}^{m, n}\left(\left.z\right|^{a_{1}, \ldots, a_{p}}\right)$ & Meijer G-function \\
\hline$\Gamma(z)$ & Gamma function \\
\hline$\Gamma(n, z)$ & Incomplete gamma function \\
\hline$\gamma$ & Euler-Mascheroni constant \\
\hline$K_{n}(z)$ & $\begin{array}{l}n \text { th-order modified Bessel function of the } \\
\text { second kind }\end{array}$ \\
\hline $\log (z)$ & Natural logarithmic function \\
\hline $\mathbb{P}\{\cdot\}$ & Probability of the enclosed random event \\
\hline$\psi_{n}(z)$ & $n$ th-order polygamma function \\
\hline$Q(z)$ & Gaussian tail function \\
\hline$U(a, b, z)$ & $\begin{array}{l}\text { Confluent hypergeometric function of the } \\
\text { second kind }\end{array}$ \\
\hline$(\cdot)^{T}$ & $\begin{array}{l}\text { Transpose operation for the enclosed } \\
\text { matrix/vector }\end{array}$ \\
\hline$(\cdot)^{H}$ & $\begin{array}{l}\text { Conjugate transpose operation for the } \\
\text { enclosed matrix/vector }\end{array}$ \\
\hline$(\cdot)^{-1}$ & $\begin{array}{l}\text { Inverse operation for the enclosed } \\
\text { matrix/vector }\end{array}$ \\
\hline$(\cdot)^{*}$ & Complex conjugate of the enclosed scalar \\
\hline$\lfloor\cdot\rfloor$ & $\begin{array}{l}\text { Floor function that rounds the enclosed } \\
\text { argument to the nearest smaller integer }\end{array}$ \\
\hline
\end{tabular}

form and all analytical results are corroborated by simulation results produced by Monte Carlo methods. Besides, we also provide comprehensive comparisons among the proposed $\mathrm{AF}$ relay assisted OFDM-IM scheme and a number of benchmarks through numerical results.

The remainder of this paper is arranged as follows. In Section II, we detail the system framework and the fundamental principle of FG AF relaying. Following these, we provide systematic performance analysis regarding average outage probability, BLER and achievable rate with a generic fixed amplification gain in Section III. To verify the analysis presented, we conduct numerical simulations according to Monte Carlo methods, and present and discuss the simulation results in Section IV, by which we can also explore a series of key features of the proposed system. Finally, we conclude the paper and point out several promising research directions that are worth studying as future work in Section $\mathrm{V}^{1}$.

\section{System Model}

\section{A. System Framework and Assumptions}

For simplicity, we suppose a typical three-node two-hop cooperative AF network consisting of one source, one FG AF relay and one destination in this paper. The FG AF relay receives the transmit OFDM block from the source, amplifies it by a fixed amplification gain, and then forwards the amplified signal to the destination. To further simplify the analysis and

\footnotetext{
${ }^{1}$ Table I lists the functions and notations involved in the performance analysis in this paper.
}

be focused, we suppose that the direct source-to-destination transmission link can not be maintained because of obstacles, heavy shadowing and/or overlong signal propagation distance. Further assuming that a half-duplex forwarding strategy is adopted, a complete transmission from source to destination via the FG AF relay thus requires two orthogonal phases. It is also assumed that a sufficiently long cyclic prefix (CP) is used so as to produce $N$ independent subcarriers. The set of these $N$ subcarriers is denoted as $\mathcal{N}$ and $|\mathcal{N}|=N$. Among these $N$ subcarriers, $T$ of them will be activated to form a SAP to convey information represented by data symbols plus extra information represented by the indices of active subcarriers. We assume CSI to be perfectly accessible without any delay at the destination only for signal detection purposes.

\section{B. Transmission, Relaying and Reception}

For information transmission, we first need to specify the mapping relation between a sequence of bits to a specific form. Different from traditional OFDM that only relies on the symbols carried on subcarriers, OFDM-IM encodes an incoming bit sequence to the SAP and the data symbols carried on active subcarriers. Specifically, a complete mapping relation consists of two interconnected parts, i.e., the mapping relation between heading bit sequence and SAP as well as the mapping relation between subsequent bit sequence and data symbols ${ }^{2}$.

As we mentioned above, a complete SAP consists of $N$ subcarrier activation states pertaining to $N$ subcarriers. Considering the application scenarios where the intermediate relay node can neither decode the received signals nor even estimate how many subcarriers are activated, we abandon the on-off keying (OOK) mapping protocol with a variable number of active subcarriers as adopted for DF relaying in [23][25]. Alternatively, we resort to the conventional look-up table mapping method with a fixed number of active subcarriers $T$ $(1 \leq T<N)$, which is proposed in [5]. The merit of the lookup table mapping method is that the AF relay node always knows the number of active subcarriers $T$ as a preset system configuration. Note that, the value of $T$ is related to the wellknown performance-rate trade-off and one should consider the practical conditions and required performance when setting $T$.

Following this setup, it is obvious that there exist $K=\left(\begin{array}{l}N \\ T\end{array}\right)$ SAPs for use. However, since binary information is intended to be transmitted (i.e., a bit stream consisting of ' 0 ' and ' 1 '), we only require a shrunk subset of $\tilde{K}=2^{\left\lfloor\log _{2}\left(\begin{array}{c}N \\ K\end{array}\right)\right\rfloor}$ SAPs, denoted by $\tilde{\mathcal{K}}=\{1,2, \ldots, \tilde{K}\}$, where $\lfloor\cdot\rfloor$ is the floor function that rounds the enclosed argument to the nearest smaller integer. The shrinking process can be achieved by various approaches, e.g., arbitrary assignment, combinatorial assignment and lexicographic assignment [5], [11], [16]. To simplify the system and avoid requiring CSI, we adopt the arbitrary assignment for the look-up table mapping as proposed in [5]. For each SAP $k \in \tilde{\mathcal{K}}$, we have the subset of $T$ active subcarriers, denoted as $\mathcal{T}(k)$. A simplistic example can be reproduced from [5] to illustrate the procedure of mapping a two-bit heading sequence (i.e., $[0,0],[0,1],[1,0]$, and $[1,1]$ ) to a

\footnotetext{
${ }^{2}$ For analytical simplicity, it is stipulated that all incoming bits are equiprobable.
} 
SAP, given $\{N, T\}=\{4,2\}$. When there are four subcarriers in total and two of them are activated to transmit, there are $\left(\begin{array}{l}4 \\ 2\end{array}\right)=6$ unique SAPs, which can be expressed as $\{1,2\}$, $\{1,3\},\{1,4\},\{2,3\},\{2,4\}$, and $\{3,4\}$. Because without CSI at the source, all subcarriers are statistically equivalent, we then arbitrarily select SAPs $\{1,2\},\{2,3\},\{3,4\}$, and $\{1,4\}$ that are mapped to the two-bit heading sequences $[0,0],[0,1]$, $[1,0]$, and $[1,1]$, respectively.

To fully exploit these $T$ active subcarriers and enhance the spectrum efficiency, we adopt the multiplexing scheme in the proposed system. The multiplexing scheme regulates that different data symbols are allowed to be carried on different active subcarriers [43]. In virtue of constant envelope [44], $M$-PSK is utilized as the amplitude-phase modulation (APM) scheme for mapping the subsequent bit sequence to data symbols. The set of $M$-ary data symbols is denoted as $\mathcal{M}$.

In order to represent a specific SAP $k$ and the data symbols carried on the active subcarriers, an OFDM block for transmission can be generated by an $N$-point inverse fast Fourier transform (IFFT), which can be written as

$$
\mathbf{x}(k)=\left[x\left(m_{1}, 1\right), x\left(m_{2}, 2\right), \ldots, x\left(m_{N}, N\right)\right]^{T} \in \mathbb{C}^{N \times 1},
$$

where $m_{n} \in \mathcal{M}$ denotes the index of data symbol carried on the $n$th subcarrier; the entry of the OFDM block $\mathbf{x}(k)$ is given by

$$
x\left(m_{n}, n\right)= \begin{cases}\chi_{m_{n}}, & n \in \mathcal{T}(k) \\ 0, & \text { otherwise }\end{cases}
$$

where $\chi_{m_{n}}$ is a normalized $M$-ary data symbol carried by the $n$th subcarrier to transmit the subsequent bit sequence, and we hereby have $\chi_{m_{n}} \chi_{m_{n}}^{*}=1$.

As a result, the received OFDM block at the AF relay can be expressed as [7]

$$
\begin{aligned}
\mathbf{y}_{1}(k) & =\left[y_{1}\left(m_{1}, 1\right), y_{1}\left(m_{2}, 2\right), \ldots, y_{1}\left(m_{N}, N\right)\right]^{T} \\
& =\sqrt{\frac{P_{t}}{T}} \mathbf{H}_{1} \mathbf{x}(k)+\mathbf{w}_{1} \in \mathbb{C}^{N \times 1},
\end{aligned}
$$

where $\mathbf{w}_{i}=\left[w_{i}(1), w_{i}(2), \ldots, w_{i}(N)\right]^{T} \in \mathbb{C}^{N \times 1}$ is the vector of $N$ complex additive white Gaussian noise (AWGN) terms on each subcarrier in the $i$ th hop, whose $N$ entries are independent and identically distributed (i.i.d.) with the noise power $N_{0} ; P_{t}$ is a uniformly distributed transmit power over $T$ active subcarriers at the source node; $\mathbf{H}_{i}=\operatorname{diag}\left\{h_{i}(1), h_{i}(2), \ldots, h_{i}(N)\right\} \in \mathbb{C}^{N \times N}$ is an $N \times N$ diagonal channel state matrix of the $i$ th hop in the frequency domain, and each diagonal entry $h_{i}(n)$ is the frequencydomain channel coefficient of the $n$th subcarrier. In this paper, we assume that all wireless channel fading complies with the frequency-flat Rayleigh fading model, and thereby the channel power gains $G_{i}(n)=\left|h_{i}(n)\right|^{2}, n \in \mathcal{N}$ are independently and exponentially distributed with mean $\mu_{i}$ in the $i$ th hop. As a result, the probability density function (PDF) $f_{G_{i}}(\zeta)$ and the cumulative distribution function (CDF) $F_{G_{i}}(\zeta)$ of the channel power gain $G_{i}(n), \forall n \in \mathcal{N}$ are given by

$$
f_{G_{i}}(\zeta)=\exp \left(-\zeta / \mu_{i}\right) / \mu_{i} \Leftrightarrow F_{G_{i}}(\zeta)=1-\exp \left(-\zeta / \mu_{i}\right) .
$$

After receiving $\mathbf{y}_{1}(k)$, the $\mathrm{FG} \mathrm{AF}$ relay with a preprocessing capability first performs a series of pre-processing operations to remove and re-insert $\mathrm{CP}$ and then simply amplifies the received signal without decoding it, and finally produces the re-transmitted signal as

$$
\mathbf{z}(k)=\alpha_{F} \mathbf{y}_{1}(k),
$$

where $\alpha_{F}$ is a preset fixed amplification gain, regardless of the adopted SAP and instantaneous CSI.

Similarly as at the relay, the received OFDM block at the destination can be expressed as ${ }^{3}$

$$
\begin{aligned}
\mathbf{y}_{2}(k) & =\left[y_{2}\left(m_{1}, 1\right), y_{2}\left(m_{2}, 2\right), \ldots, y_{2}\left(m_{N}, N\right)\right]^{T} \\
& =\mathbf{H}_{2} \mathbf{z}(k)+\mathbf{w}_{2} \\
& =\alpha_{F} \sqrt{\frac{P_{t}}{T}} \mathbf{H}_{1} \mathbf{H}_{2} \mathbf{x}(k)+\underbrace{\alpha_{F} \mathbf{H}_{2} \mathbf{w}_{1}+\mathbf{w}_{2}}_{\mathbf{w}_{\Sigma}},
\end{aligned}
$$

where $\mathbf{w}_{\Sigma}=\left[w_{\Sigma}(1), w_{\Sigma}(2), \ldots, w_{\Sigma}(N)\right]^{T} \in \mathbb{C}^{N \times 1}$ denotes the vector of $N$ aggregate complex AWGN terms at the destination. The entry $w_{\Sigma}(n)$ of $\mathbf{w}_{\Sigma}$ follows the distribution $\mathcal{C N}\left(0,\left(\alpha_{F}^{2} G_{2}(n)+1\right) N_{0}\right)$, which is obviously not completely independent, rather associated with instantaneous channel state $h_{2}(n)$ of the second hop.

According to (6), the end-to-end received signal-to-noise ratio (SNR) for the $n$th active subcarrier (i.e., $n \in \mathcal{T}(k)$ ) at the destination is determined by

$$
\operatorname{SNR}(k, n)=\frac{\alpha_{F}^{2} G_{1}(n) G_{2}(n) P_{t}}{\left(\alpha_{F}^{2} G_{2}(n)+1\right) T N_{0}} .
$$

Then, by processing and decoding $\mathbf{y}_{2}(k)$ according to the maximum-likelihood (ML) estimation, the transmit OFDM block $\mathbf{x}(k)$ can be detected, from which the complete transmitted bit sequence from the source including both heading and subsequent bit sequences can be finally retrieved at the destination. The ML detection criterion specially tailored for AF relay networks contaminated by colored noise is given infra [48]:

$$
\begin{aligned}
\hat{\mathbf{x}}(\hat{k})=\underset{\mathbf{x}(k) \in \mathcal{X}}{\arg \min }[ & \left(\dot{\mathbf{y}}_{2}(\dot{k})-\alpha_{F} \sqrt{\frac{P_{t}}{T}} \mathbf{H}_{1} \mathbf{H}_{2} \mathbf{x}(k)\right)^{H} \mathbf{\Lambda}^{-\mathbf{1}} \\
& \left.\times\left(\dot{\mathbf{y}}_{2}(\dot{k})-\alpha_{F} \sqrt{\frac{P_{t}}{T}} \mathbf{H}_{1} \mathbf{H}_{2} \mathbf{x}(k)\right)\right],
\end{aligned}
$$

where $\dot{\mathbf{y}}_{2}(\dot{k})$ denotes the actual received OFDM block at the destination that has been contaminated by noise; $\mathbf{x}(k)$ denotes the estimation trial; the full set of all possible $\mathbf{x}(k)$ is denoted as $\mathcal{X}$, and obviously, its cardinality $|\mathcal{X}|=2^{\left\lfloor\log _{2}\left(\begin{array}{c}N \\ T\end{array}\right)\right\rfloor} M^{T}$ characterizes the size of search space; $\hat{\mathbf{x}}(\hat{k})$ denotes the estimated transmit OFDM block at the destination; $\boldsymbol{\Lambda}$ is the equivalent

\footnotetext{
${ }^{3}$ In this paper, we assume that the FG AF relay node is capable of performing some pre-processing operations prior to amplifying and re-transmitting received signals, which include $\mathrm{CP}$ removal and re-insertion. This is a common assumption adopted in existing literature to reduce the CP length and enhance end-to-end transmission efficiency [45]-[47]. This assumption allows the fast Fourier transform (FFT) to yield a diagonal channel state matrix that multiplies $\mathbf{w}_{1}$, and the end-to-end received signal block can thereby be expressed in the form of (6).
} 
noise covariance matrix having a diagonal structure with not necessarily equal diagonal elements, and can be explicitly written as

$$
\begin{gathered}
\boldsymbol{\Lambda}=\mathbb{E}\left\{\mathbf{w}_{\Sigma} \mathbf{w}_{\Sigma}^{H}\right\} \in \mathbb{C}^{N \times N} \\
=\operatorname{diag}\left\{\left(\alpha_{F}^{2} G_{2}(1)+1\right) N_{0},\left(\alpha_{F}^{2} G_{2}(2)+1\right) N_{0},\right. \\
\left.\ldots,\left(\alpha_{F}^{2} G_{2}(N)+1\right) N_{0}\right\} .
\end{gathered}
$$

\section{PERformance AnAlysis}

\section{A. Preliminaries of Performance Evaluation Metrics}

Here we define three important performance evaluation metrics that can be used to evaluate different aspects of the proposed FG AF relay assisted OFDM-IM system.

1) Average outage probability: In accordance with the definition of an end-to-end outage event for multi-hop multicarrier systems in [49], we define the outage event of the proposed FG AF relay assisted OFDM-IM system infra:

Definition 1: An outage event occurs once the end-to-end received SNR at the destination of any active subcarrier (i.e., (7)) falls behind a preset outage threshold $s$.

According to Definition 1, the conditional end-to-end outage probability on the SAP $k$ can be determined by

$$
P_{o}(s \mid k)=\mathbb{P}\left\{\bigcup_{n \in \mathcal{T}(k)}\{\operatorname{SNR}(k, n)<s\}\right\} .
$$

Moreover, to consider the average situation over all $\tilde{K}$ SAPs, we average the conditional outage probability $P_{o}(s \mid k)$ given above over SAP $k \in \tilde{\mathcal{K}}$ and hereby derive the average outage probability as:

$$
\bar{P}_{o}(s)=\underset{k \in \tilde{\mathcal{K}}}{\mathbb{E}}\left\{P_{o}(s \mid k)\right\} .
$$

The average outage probability indicates the quality of endto-end signal detection of FG AF relay assisted OFDM-IM systems.

2) Average BLER: In a similar manner, we can ditto regard the block error event from an end-to-end perspective. The conditional BLER on transmit OFDM block $\dot{\mathrm{x}}(\dot{k})$, and CSI can therefore be approximated by the union bound as

$$
P_{e}\left(\dot{\mathbf{x}}(\dot{k}) \mid \mathbf{H}_{1}, \mathbf{H}_{2}\right) \approx \sum_{\hat{\mathbf{x}}(\hat{k}) \neq \dot{\mathbf{x}}(\dot{k})} P_{e}\left(\dot{\mathbf{x}}(\dot{k}) \rightarrow \hat{\mathbf{x}}(\hat{k}) \mid \mathbf{H}_{1}, \mathbf{H}_{2}\right)
$$

where $P_{e}\left(\dot{\mathbf{x}}(\dot{k}) \rightarrow \hat{\mathbf{x}}(\hat{k}) \mid \mathbf{H}_{1}, \mathbf{H}_{2}\right)$ denotes the conditional probability of the block error event (i.e., the original transmit block $\dot{\mathbf{x}}(\dot{k})$ at the source is erroneously estimated to $\hat{\mathbf{x}}(\hat{k})$ at the destination), given $\mathbf{H}_{1}$ and $\mathbf{H}_{2}$. Subsequently, we can average the conditional BLER over transmit OFDM block $\dot{\mathbf{x}}(\dot{k})$ and channel state matrices $\mathbf{H}_{1}, \mathbf{H}_{2}$ to determine the average BLER as

$$
\bar{P}_{e}=\underset{\dot{\mathbf{x}}(\dot{k}) \in \mathcal{X}, \mathbf{H}_{1}, \mathbf{H}_{2}}{\mathbb{E}}\left\{P_{e}\left(\dot{\mathbf{x}}(\dot{k}) \mid \mathbf{H}_{1}, \mathbf{H}_{2}\right)\right\},
$$

which implies the end-to-end fidelity of FG AF relay assisted OFDM-IM systems.
3) Average achievable rate: Except for detection quality and fidelity, throughput is another crucial performance evaluation metric characterizing the transmission efficiency of the proposed FG AF relay assisted OFDM-IM system. For multihop cooperative networks, we can resort to the max-flow mincut theorem and define the conditional achievable rate on SAP $k$ and channel state matrices $\mathbf{H}_{1}, \mathbf{H}_{2}$ as [50]

$$
C\left(k \mid \mathbf{H}_{1}, \mathbf{H}_{2}\right)=\frac{1}{2} \sum_{n \in \mathcal{T}(k)} \log (1+\operatorname{SNR}(k, n)),
$$

where the presence of multiplying factor $1 / 2$ is due to the requirement of two orthogonal phases for one complete transmission from source to destination by half-duplex relaying.

To reflect the average situation, we average the conditional achievable rate $C\left(k \mid \mathbf{H}_{1}, \mathbf{H}_{2}\right)$ over SAP $k$ and channel state matrices $\mathbf{H}_{1}, \mathbf{H}_{2}$ and obtain the average achievable rate by

$$
\bar{C}=\underset{k \in \tilde{\mathcal{K}}, \mathbf{H}_{1}, \mathbf{H}_{2}}{\mathbb{E}}\left\{C\left(k \mid \mathbf{H}_{1}, \mathbf{H}_{2}\right)\right\},
$$

which can evaluate the end-to-end throughput of FG AF relay assisted OFDM-IM systems.

\section{B. Outage Performance Analysis}

Because all subcarriers are assumed to be mutually independent in OFDM-IM systems with a sufficiently long CP, we can view the outage event in a subcarrier-wise manner. For an arbitrary active subcarrier, we can derive the subcarrier-wise conditional outage probability as [32]

$$
\begin{aligned}
& \Phi(s \mid k)=\mathbb{P}\{\operatorname{SNR}(k, n)<s\} \\
& =1-\frac{2}{\alpha_{F}} \sqrt{\frac{s T N_{0}}{P_{t} \mu_{1} \mu_{2}}} \exp \left(-\frac{s T N_{0}}{P_{t} \mu_{1}}\right) K_{1}\left(\frac{2}{\alpha_{F}} \sqrt{\frac{s T N_{0}}{P_{t} \mu_{1} \mu_{2}}}\right),
\end{aligned}
$$

where $K_{n}(\cdot)$ represents the $n$ th-order modified Bessel function of the second kind.

By order statistics and (16), we can easily obtain the subcarrier-wise conditional outage probability of the $\xi$ th smallest SNR by

$$
\Phi_{(\xi)}(s \mid k)=\sum_{n=\xi}^{N}\left(\begin{array}{l}
N \\
n
\end{array}\right)(\Phi(s \mid k))^{n}(1-\Phi(s \mid k))^{N-n} .
$$

If there exist $T$ active subcarriers selected from total $N$ subcarriers, the worst subcarrier (i.e., the subcarrier with the lowest end-to-end SNR) is the $\xi$ th worst, and the range of $\xi$ is given by $1 \leq \xi \leq N-T+1$. Consequently, according to Lemma 1 proven in [20], the conditional outage probability on $\mathrm{SAP} k$ is given by

$$
P_{o}(s \mid k)=\sum_{\xi=1}^{N-T+1} \Upsilon(k, \xi) \Phi_{(\xi)}(s \mid k),
$$

where

$$
\Upsilon(k, \xi)=\left(\begin{array}{l}
N-\xi \\
T-1
\end{array}\right) /\left(\begin{array}{l}
N \\
T
\end{array}\right)
$$

is the conditional occurrence probability of the event that the worst subcarrier is the $\xi$ th worst. 
Since the heading bit sequence has a fixed length and is equiprobable, all $\tilde{K}$ SAPs will occur with the same probability. Also because all SAPs yield the same number of active subcarriers $T$, they can be regarded as statistically equivalent when considering outage performance. By these two properties, we can thereby remove the conditional dependence on SAP $k$ and derive the final expression of average outage probability in (20) at the top of the next page. An important note observed from (20) is that the outage performance of the proposed system can only be affected by the number of active subcarriers $T$, instead of the number of total subcarriers $N$.

\section{Error Performance Analysis}

Because the FG AF relay does not need to decode the received signal, the estimation error will only take place at the destination when AF relaying is applied. To derive the average BLER, we can first dedicate to the fundamental element $P_{e}\left(\dot{\mathbf{x}}(\dot{k}) \rightarrow \hat{\mathbf{x}}(\hat{k}) \mid \mathbf{H}_{1}, \mathbf{H}_{2}\right)$, i.e., the conditional pairwise error probability that the original transmit block $\dot{\mathbf{x}}(\dot{k})$ at the source is erroneously estimated to $\hat{\mathbf{x}}(\hat{k})$ at the destination. By basic theories of the ML detection, we can express $P_{e}(\dot{\mathbf{x}}(\dot{k}) \rightarrow$ $\left.\hat{\mathbf{x}}(\hat{k}) \mid \mathbf{H}_{1}, \mathbf{H}_{2}\right)$ by the Gaussian tail function as [24]

$$
\begin{aligned}
& P_{e}\left(\dot{\mathbf{x}}(\dot{k}) \rightarrow \hat{\mathbf{x}}(\hat{k}) \mid \mathbf{H}_{1}, \mathbf{H}_{2}\right) \\
& =Q\left(\sqrt{\frac{\alpha_{F}^{2} P_{t}}{T N_{0}} \sum_{n=1}^{N} \frac{G_{1}(n) G_{2}(n) \Delta\left(n, \dot{k}, \hat{k}, \dot{m}_{n}, \hat{m}_{n}\right)}{\alpha_{F}^{2} G_{2}(n)+1}}\right)
\end{aligned}
$$

where $Q(z)=\frac{1}{\sqrt{2 \pi}} \int_{z}^{\infty} \exp \left(-\frac{u^{2}}{2}\right) \mathrm{d} u$ denotes the Gaussian tail function and

$$
\Delta\left(n, \dot{k}, \hat{k}, \dot{m}_{n}, \hat{m}_{n}\right)=\left|\dot{x}\left(\dot{m}_{n}, n\right)-\hat{x}\left(\hat{m}_{n}, n\right)\right|^{2} .
$$

However, the Gaussian tail function is mathematically intractable, which makes further analysis difficult. In order to provide insightful analysis of BLER, we adopt the following approximation of the Gaussian tail function in order to simplify the analysis in the sequel [5]:

$$
Q(z) \approx \frac{1}{12} \exp \left(-\frac{z^{2}}{2}\right)+\frac{1}{4} \exp \left(-\frac{2 z^{2}}{3}\right)
$$

which is accurate for large $z$. By (23), we can approximate $P_{e}\left(\dot{\mathbf{x}}(\dot{k}) \rightarrow \hat{\mathbf{x}}(\hat{k}) \mid \mathbf{H}_{1}, \mathbf{H}_{2}\right)$ to be

$$
\begin{aligned}
& P_{e}\left(\dot{\mathbf{x}}(\dot{k}) \rightarrow \hat{\mathbf{x}}(\hat{k}) \mid \mathbf{H}_{1}, \mathbf{H}_{2}\right) \\
& \approx \frac{1}{12} \exp \left(-\frac{\alpha_{F}^{2} P_{t}}{2 T N_{0}} \sum_{n=1}^{N} \frac{G_{1}(n) G_{2}(n) \Delta\left(n, \dot{k}, \hat{k}, \dot{m}_{n}, \hat{m}_{n}\right)}{\alpha_{F}^{2} G_{2}(n)+1}\right) \\
& +\frac{1}{4} \exp \left(-\frac{2 \alpha_{F}^{2} P_{t}}{3 T N_{0}} \sum_{n=1}^{N} \frac{G_{1}(n) G_{2}(n) \Delta\left(n, \dot{k}, \hat{k}, \dot{m}_{n}, \hat{m}_{n}\right)}{\alpha_{F}^{2} G_{2}(n)+1}\right) .
\end{aligned}
$$

Subsequently, according to the basic property of the exponential function: $\exp \left(\sum_{n} x_{n}\right)=\prod_{n} \exp \left(x_{n}\right)$, we can rewrite (24) as

$$
\begin{aligned}
& P_{e}\left(\dot{\mathbf{x}}(\dot{k}) \rightarrow \hat{\mathbf{x}}(\hat{k}) \mid \mathbf{H}_{1}, \mathbf{H}_{2}\right) \\
& \approx \frac{1}{12} \prod_{n=1}^{N} \exp \left(-\frac{\alpha_{F}^{2} P_{t} G_{1}(n) G_{2}(n) \Delta\left(n, \dot{k}, \hat{k}, \dot{m}_{n}, \hat{m}_{n}\right)}{2\left(\alpha_{F}^{2} G_{2}(n)+1\right) T N_{0}}\right) \\
& +\frac{1}{4} \prod_{n=1}^{N} \exp \left(-\frac{2 \alpha_{F}^{2} P_{t} G_{1}(n) G_{2}(n) \Delta\left(n, \dot{k}, \hat{k}, \dot{m}_{n}, \hat{m}_{n}\right)}{3\left(\alpha_{F}^{2} G_{2}(n)+1\right) T N_{0}}\right) .
\end{aligned}
$$

Now, we take advantage of the independence among subcarriers and remove the conditions on $P_{e}\left(\dot{\mathbf{x}}(\dot{k}) \rightarrow \hat{\mathbf{x}}(\hat{k}) \mid \mathbf{H}_{1}, \mathbf{H}_{2}\right)$ in (26) at the top of the next page, where $\left\{\omega_{1}, \omega_{2}\right\}=\{1 / 12,1 / 4\}$ and $\left\{\lambda_{1}, \lambda_{2}\right\}=\{1 / 2,2 / 3\} ; \mathcal{E}(z)=\exp (z) \operatorname{Ei}(-z)$ and $\operatorname{Ei}(z)=\int_{z}^{\infty} \frac{e^{-t}}{t} \mathrm{~d} t$ is the exponential integral function.

As a result, the unconditional BLER when transmitting $\dot{\mathbf{x}}(\dot{k})$ can be derived by

$$
\begin{aligned}
P_{e}(\dot{\mathbf{x}}(\dot{k})) & =\underset{\mathbf{H}_{1}, \mathbf{H}_{2}}{\mathbb{E}}\left\{P_{e}\left(\dot{\mathbf{x}}(\dot{k}) \mid \mathbf{H}_{1}, \mathbf{H}_{2}\right)\right\} \\
& \stackrel{(a)}{\approx} \sum_{\hat{\mathbf{x}}(\hat{k}) \neq \dot{\mathbf{x}}(\dot{k})} \underset{\mathbf{H}_{1}, \mathbf{H}_{2}}{\mathbb{E}}\left\{P_{e}\left(\dot{\mathbf{x}}(\dot{k}) \rightarrow \hat{\mathbf{x}}(\hat{k}) \mid \mathbf{H}_{1}, \mathbf{H}_{2}\right)\right\} \\
& =\sum_{\hat{\mathbf{x}}(\hat{k}) \neq \dot{\mathbf{x}}(\dot{k})} P_{e}(\dot{\mathbf{x}}(\dot{k}) \rightarrow \hat{\mathbf{x}}(\hat{k})),
\end{aligned}
$$

where $(a)$ is valid because of the union bound and the interchangeability between expectation and summation operations.

Finally, the average BLER can be determined by averaging over all possible transmit OFDM blocks $\dot{\mathbf{x}}(\dot{k}) \in \mathcal{X}$ as

$$
\bar{P}_{e}=\frac{1}{|\mathcal{X}|} \sum_{\dot{\mathbf{x}}(\dot{k}) \in \mathcal{X}} P_{e}(\dot{\mathbf{x}}(\dot{k})) \text {. }
$$

\section{Achievable Rate Analysis}

To investigate the average achievable rate, we consider $\operatorname{SNR}(k, n)$ as an independent random variable and $\Phi(s \mid k)$ as its CDF. Based on the $\operatorname{CDF}$ of $\operatorname{SNR}(k, n)$ given in (16), we then derive the PDF in (29) at the top of the next page. Therefore, the unconditional achievable rate $C\left(k \mid \mathbf{H}_{1}, \mathbf{H}_{2}\right)$ can be obtained by averaging $C\left(k \mid \mathbf{H}_{1}, \mathbf{H}_{2}\right)$ over $\operatorname{SNR}(k, n)$ as

$$
\begin{aligned}
C(k) \stackrel{(a)}{=} \frac{1}{2} \sum_{n \in \mathcal{T}(k)} \int_{0}^{\infty} \log (1+\operatorname{SNR}(k, n)) \phi & \operatorname{SNR}(k, n) \mid k) \\
& \times \operatorname{dSNR}(k, n),
\end{aligned}
$$

where $(a)$ is valid owing to the interchangeability between summation and integration operations as well as the independence among all $N$ subcarriers.

However, to the best of authors' knowledge, the closedform expression of (30) does not exist. To investigate the relation among achievable rate and key parameters, we assume $\operatorname{SNR}(k, n) \gg 1$ and approximate $C(k)$ in closed form in (31) at the top of the next page, where $A=\alpha_{F}^{2} \mu_{2}$ and $B=\frac{T N_{0}}{P_{t} \mu_{1}} ; \gamma$ is the Euler-Mascheroni constant; $\Gamma(n, z)=\int_{z}^{\infty} t^{n-1} \exp (-t)$ 


$$
\bar{P}_{o}(s)=\underset{k \in \tilde{\mathcal{K}}}{\mathbb{E}}\left\{P_{o}(s \mid k)\right\}=1-\left(\frac{2}{\alpha_{F}} \sqrt{\frac{s T N_{0}}{P_{t} \mu_{1} \mu_{2}}} \exp \left(-\frac{s T N_{0}}{P_{t} \mu_{1}}\right) K_{1}\left(\frac{2}{\alpha_{F}} \sqrt{\frac{s T N_{0}}{P_{t} \mu_{1} \mu_{2}}}\right)\right)^{T}
$$

$$
\begin{aligned}
& P_{e}(\dot{\mathbf{x}}(\dot{k}) \rightarrow \hat{\mathbf{x}}(\hat{k}))=\underset{\mathbf{H}_{1}, \mathbf{H}_{2}}{\mathbb{E}}\left\{P_{e}\left(\dot{\mathbf{x}}(\dot{k}) \rightarrow \hat{\mathbf{x}}(\hat{k}) \mid \mathbf{H}_{1}, \mathbf{H}_{2}\right)\right\} \\
& \approx \sum_{i=1}^{2} \omega_{i} \prod_{n=1}^{N} \int_{0}^{\infty} \int_{0}^{\infty} \exp \left(-\frac{\lambda_{i} \alpha_{F}^{2} P_{t} G_{1}(n) G_{2}(n) \Delta\left(n, \dot{k}, \hat{k}, \dot{m}_{n}, \hat{m}_{n}\right)}{\left(\alpha_{F}^{2} G_{2}(n)+1\right) T N_{0}}\right) f_{G_{1}}\left(G_{1}(n)\right) f_{G_{2}}\left(G_{2}(n)\right) \mathrm{d} G_{1}(n) \mathrm{d} G_{2}(n) \\
& =\sum_{i=1}^{2} \omega_{i} \prod_{n=1}^{N}\left\{\frac{T N_{0}}{\alpha_{F}^{2} \mu_{2}\left(T N_{0}+\lambda_{i} P_{t} \mu_{1} \Delta\left(n, \dot{k}, \hat{k}, \dot{m}_{n}, \hat{m}_{n}\right)\right)^{2}}\right. \\
& \left.\times\left[\alpha_{F}^{2} \mu_{2}\left(T N_{0}+\lambda_{i} P_{t} \mu_{1} \Delta\left(n, \dot{k}, \hat{k}, \dot{m}_{n}, \hat{m}_{n}\right)\right)-\lambda_{i} P_{t} \mu_{1} \Delta\left(n, \dot{k}, \hat{k}, \dot{m}_{n}, \hat{m}_{n}\right) \mathcal{E}\left(\frac{T N_{0}}{T N_{0} \alpha_{F}^{2} \mu_{2}+\lambda_{i} \alpha_{F}^{2} P_{t} \mu_{1} \mu_{2} \Delta\left(n, \dot{k}, \hat{k}, \dot{m}_{n}, \hat{m}_{n}\right)}\right)\right]\right\}
\end{aligned}
$$

$$
\phi(s \mid k)=\frac{\mathrm{d} \Phi(s \mid k)}{\mathrm{d} s}=\frac{2 T N_{0}}{\alpha_{F}^{2} P_{t} \mu_{1} \mu_{2}} \exp \left(-\frac{s T N_{0}}{P_{t} \mu_{1}}\right)\left(K_{0}\left(\frac{2}{\alpha_{F}} \sqrt{\frac{s T N_{0}}{P_{t} \mu_{1} \mu_{2}}}\right)+\alpha_{F} \mu_{2} \sqrt{\frac{s T N_{0}}{P_{t} \mu_{1} \mu_{2}}} K_{1}\left(\frac{2}{\alpha_{F}} \sqrt{\frac{s T N_{0}}{P_{t} \mu_{1} \mu_{2}}}\right)\right)
$$

$$
\begin{aligned}
& C(k) \approx \frac{1}{2} \sum_{n \in \mathcal{T}(k)} \int_{0}^{\infty} \log (\operatorname{SNR}(k, n)) \phi(\operatorname{SNR}(k, n) \mid k) \operatorname{dSNR}(k, n) \\
& =\frac{T}{2 A}\left\{-\exp \left(\frac{1}{A}\right)\left[\Gamma\left(-1, \frac{1}{A}\right)(-1+2 \gamma+\log (B))+\Gamma\left(0, \frac{1}{A}\right)(2 \gamma+\log (B))\right]+A V\left(1,0, \frac{1}{A}\right)+V\left(1,1, \frac{1}{A}\right)\right\}
\end{aligned}
$$

is the incomplete gamma function; $V(a, b, z)=\frac{\partial U(a, b, z)}{\partial a}$ is a defined function and $U(a, b, z)=\frac{\int_{0}^{\infty} e^{-z t} t^{a-1}(1+t)^{b-a-1} \mathrm{~d} t}{\int_{0}^{\infty} t^{a-1} e^{-t} \mathrm{~d} t}$ is the confluent hypergeometric function of the second kind. For understanding (31) better, we derive the closed-form expressions of $V(a, b, z)$ for two special cases of $\{a, b\}=\{1,0\}$ and $\{a, b\}=\{1,1\}$ when $z>0$ in Appendix A.

On the other hand, if $\operatorname{SNR}(k, n) \ll 1$, we can similarly perform power series expansion on $\operatorname{SNR}(k, n)$ and approximate $\log (1+\operatorname{SNR}(k, n)) \approx \operatorname{SNR}(k, n)$, which can be substituted into (30) to yield a similar form as (31), but consisting of $V(2,2,1 / A)$ and $V(3,3,1 / A)$.

Since the heading bit sequence has a fixed length and is equiprobable, all $\tilde{K}$ SAPs will occur with the same probability and are equivalent in terms of achievable rate. Finally, we average $C(k)$ over all SAPs $\forall k \in \tilde{\mathcal{K}}$ and obtain the average achievable rate expressed by the same form as given in (31), i.e.,

$$
\bar{C}=\frac{1}{|\mathcal{X}|} \sum_{k \in \tilde{\mathcal{K}}} C(k)=C(k)
$$

\section{NUMERICAL RESUlts AND Discussion}

\section{A. Verification}

To substantiate the analytical results presented in Section III, we conducted relevant numerical simulations by Monte Carlo methods and present the simulation results in this section with appropriate discussions. Without loss of generality, we

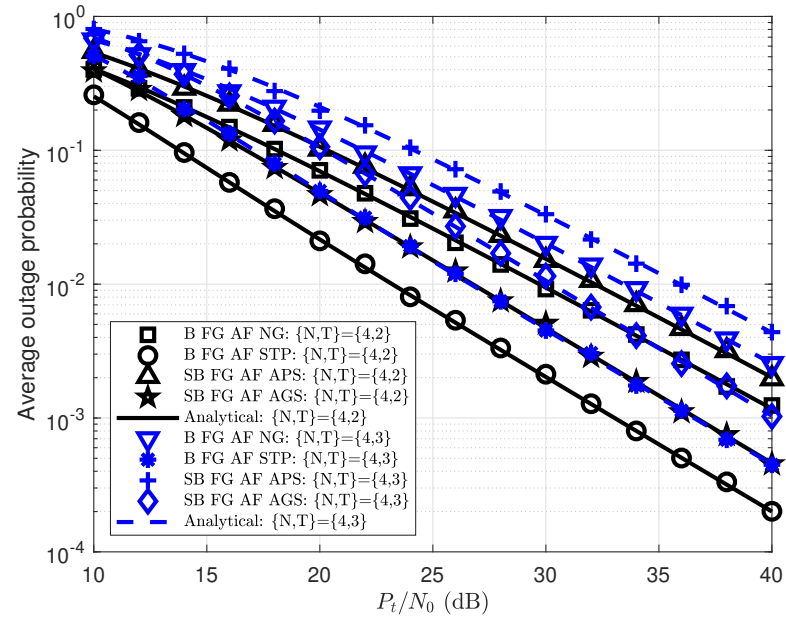

Fig. 1: Average outage probability versus ratio of transmit power to noise power $P_{t} / N_{0}$ for different FG AF relaying schemes with different parameter sets $\{N, T\}$.

normalize the outage threshold $s$ and noise power $N_{0}$, and set the average channel power gain $\mu_{1}=2$ and $\mu_{2}=2$ in all simulations. For simplicity, we further adopted binary PSK (BPSK, i.e., $M=2$ ) as the APM scheme throughout all simulations presented in this section, except the simulations associated with error performance in which both BPSK and 


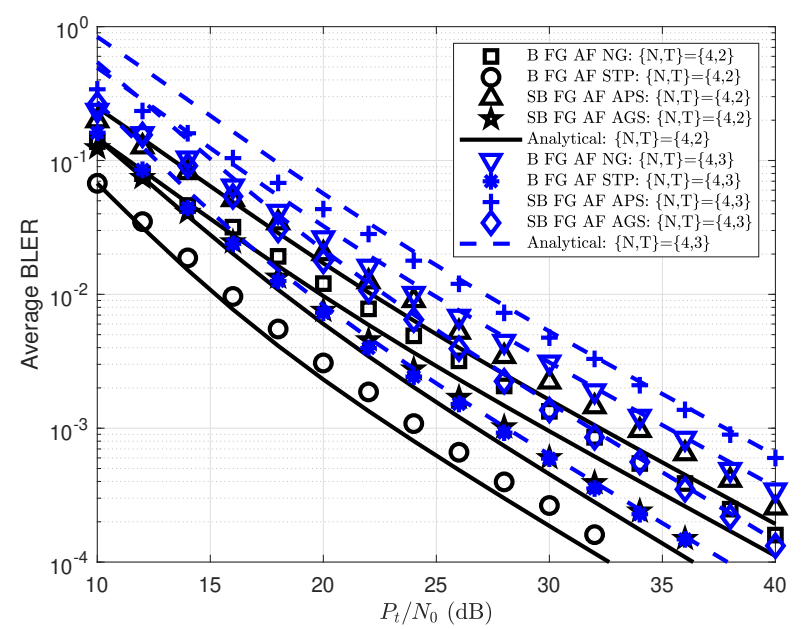

(a) BPSK

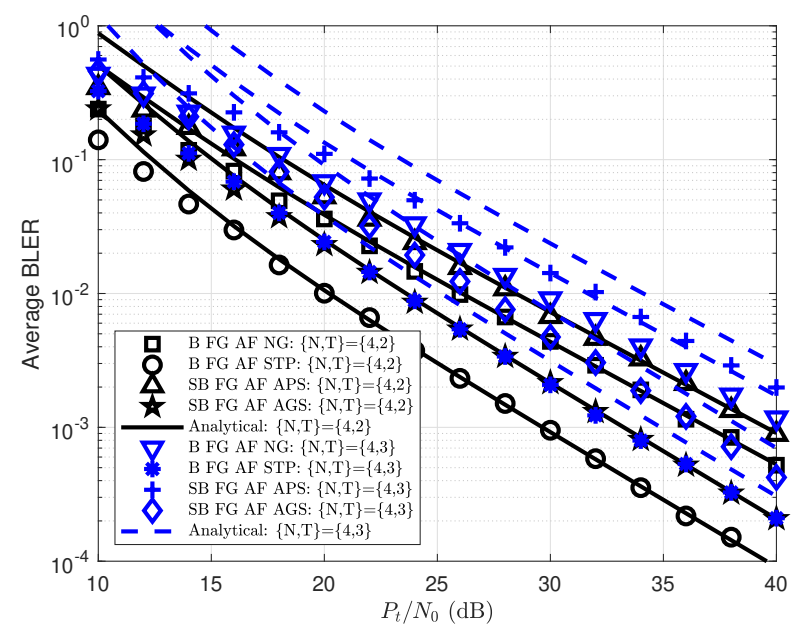

(b) QPSK

Fig. 2: Average BLER versus ratio of transmit power to noise power $P_{t} / N_{0}$ for different FG AF relaying schemes with different parameter sets $\{N, T\}$.

quadrature PSK (QPSK, i.e., $M=4$ ) are adopted. To be general, we employed several classic FG AF relaying schemes with different fixed amplification gains in the simulations, which include

- Blind FG AF relaying with normalized gain $G_{F}=1$ (B FG AF NG)

- Blind FG AF relaying with the same transmit power as the source, i.e., $G_{F}=\sqrt{\frac{P_{t}}{T}}$ (B FG AF STP)

- Semi-blind FG AF relaying with average power scaling, i.e., $G_{F}=\sqrt{\mathbb{E}\left\{\frac{P_{t} / T}{P_{t} G_{1}(n) / T+N_{0}}\right\}}$ (SB FG AF APS)

- Semi-blind FG AF relaying with average gain scaling, i.e., $G_{F}=\sqrt{\frac{P_{t} / T}{P_{t} \mu_{1} / T+N_{0}}}$ (SB FG AF AGS)

The numerical results pertaining to average outage probability, BLER and achievable rate are presented in Fig. 1, 2 and 3, respectively. From these three figures, we have the following key observations.

First of all, the correctness of all analysis presented in

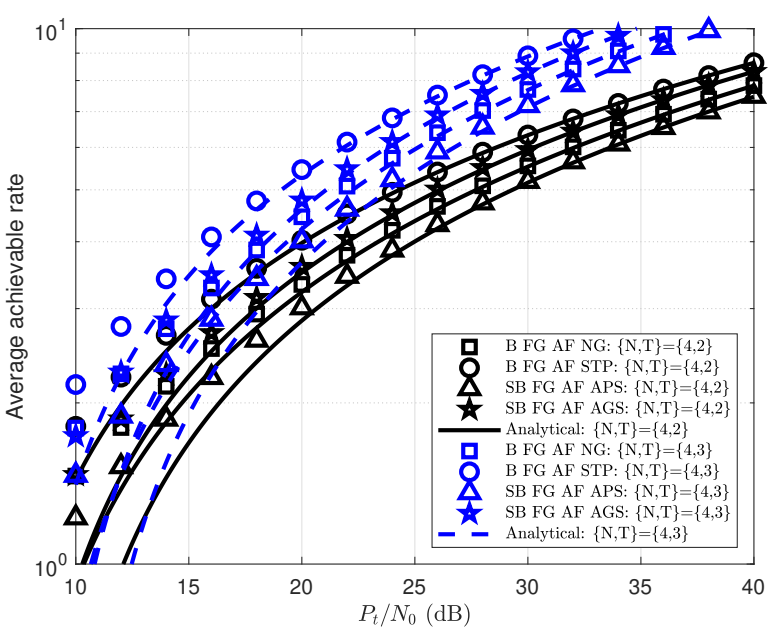

Fig. 3: Average achievable rate versus ratio of transmit power to noise power $P_{t} / N_{0}$ for different FG AF relaying schemes with different parameter sets $\{N, T\}$.

Section III has been verified by the numerical results provided. As in all cases, the analytical curves match the numerical results. As shown in Fig. 1, the analytical results perfectly match the numerical results in all cases. This indicates that (20) is the exact expression of the average outage probability instead of an approximation. On the contrary, the accuracy of (28) and (32) are affected by simulation parameters as shown in Fig. 2 and 3, especially the ratio of transmit power to noise power $P_{t} / N_{0}$, because both are approximations for large average SNR. The gap between analytical and numerical results of average BLER is caused by the approximation of the Gaussian tail function provided in (23) as well as the union bound approximation in (12). Likewise, the approximation of unconditional achievable rate $C(k)$ adopted in (31) renders the analytical-numerical gap for average achievable rate.

Also, the impacts of different simulation parameters on three performance evaluation metrics can be revealed by observing these three figures. First, it is obvious that the outage performance will be enhanced by activating a smaller number of subcarriers. This is simply because, by Definition 1, the end-to-end outage event in this paper is determined by the worst active subcarrier (i.e., the active subcarrier with the lowest received SNR at the destination). Therefore, with a smaller number of active subcarriers, it is more likely that the end-to-end SNRs of all subcarriers are larger than the preset outage threshold $s$. Second, increasing the number of active subcarriers $T$ will render a higher average BLER, since it will be more difficult to distinguish adjacent OFDM blocks by the ML estimation. Third, a larger number of subcarriers $T$ will yield a higher average achievable rate for FG AF relay assisted OFDM-IM systems, because more subcarriers are in use by the multiplexing scheme for data transmission. This refers to the well-known performance-rate trade-off in multicarrier systems.

In addition, throughout these simulation results, we can gain insights into different FG AF relaying schemes as well as their pros and cons. More specifically, there is a trade- 
off among performance, complexity and energy consumption when choosing the FG AF relaying scheme. The blind FG $\mathrm{AF}$ relaying with a normalized gain is a naïve scheme, which only suites the theoretical research for its simplicity. The blind FG AF relaying with the same transmit power as the source outperforms others, because a larger amount of transmit power is used on average. However, in practical AF relay systems when the non-linear amplification region is taken into account, this blind scheme could lead to an over-amplified signal and the non-linear amplification problem, which could thereby result in worse performance. To be subtle by making use of statistical CSI, semi-blind AF relaying schemes would be applied. There are also different ways to involve statistical CSI depending on the performance requirements and system configurations. The semi-blind FG AF relaying scheme with average gain scaling has the same average gain as the VG AF relaying scheme, while the semi-blind FG AF relaying scheme with average power scaling shares the same average channel power gain as the $\mathrm{VG}$ AF relaying scheme.

\section{B. Comparisons}

Apart from verification purposes, we also provide comparisons among the proposed FG AF relay assisted OFDM-IM and a series of performance benchmarks, including

- FG AF relay assisted classic OFDM

- DF relay assisted OFDM-IM

- VG AF relay assisted OFDM-IM with variable amplification gain $G_{V}(n)=\sqrt{\frac{P_{t} / T}{G_{1}(n) P_{t} / T+N_{0}}}$

to fully reveal the pros and cons as well as application scenarios of the proposed scheme. The numerical results regarding average outage probability, BLER and achievable rate are presented in Fig. 4, Fig. 5, and Fig. 6, respectively. To be comparable, we adopt the semi-blind FG AF relaying schemes with average power scaling and average gain scaling for the FG AF relay assisted OFDM-IM and classic OFDM systems.

From these three figures, we first observe the trade-off between reliability and achievable rate. Although the relayed OFDM-IM system outperforms the relayed classic OFDM system in terms of outage and error performance, as the number of active subcarriers declines, the relayed OFDMIM system owns a lower achievable rate. Another trade-off between performance and complexity can also be observed. With a powerful channel estimation module, a relay node is able to obtain more CSI so as to yield better performance. Since a DF relay is capable of estimating the instantaneous CSI and decoding the received signal, DF relay assisted OFDMIM has the best performance over the other cases. Similarly, VG AF relaying requiring the instantaneous CSI without decoding the received signal provides worse performance than DF relaying, while is still better than FG AF relaying that has no instantaneous CSI.

\section{CONCLUSiOn AND Future WORK}

To enhance the practicality of relay assisted OFDM-IM, we proposed the FG AF relay assisted OFDM-IM system in

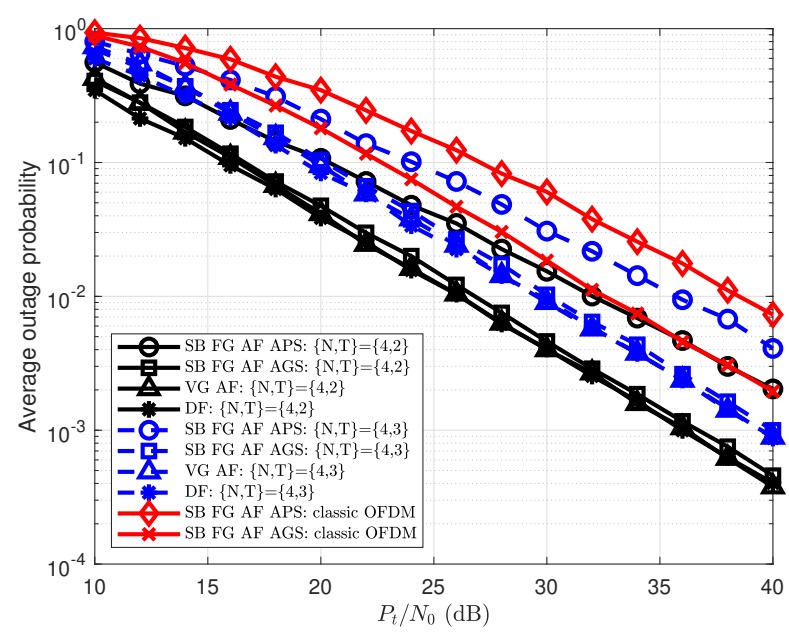

Fig. 4: Comparison of average outage probability among different multi-carrier relay systems.

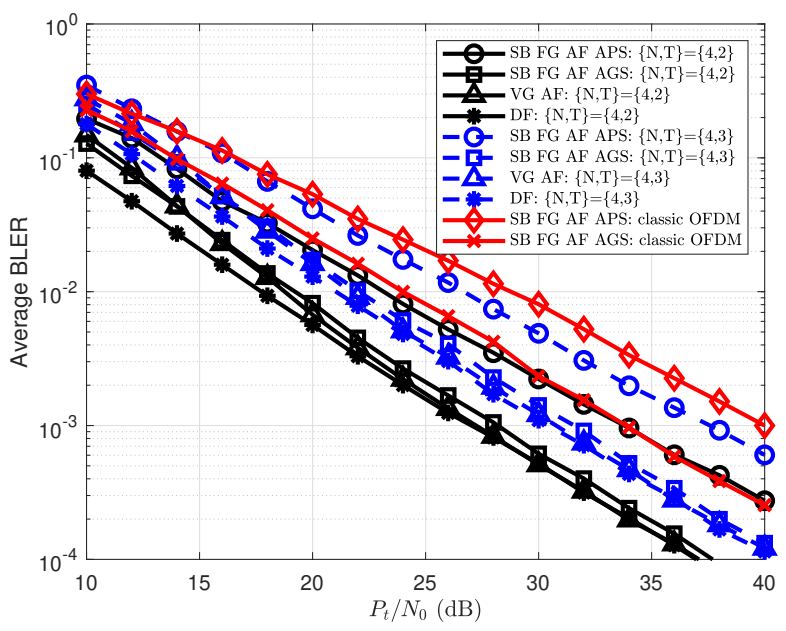

(a) BPSK

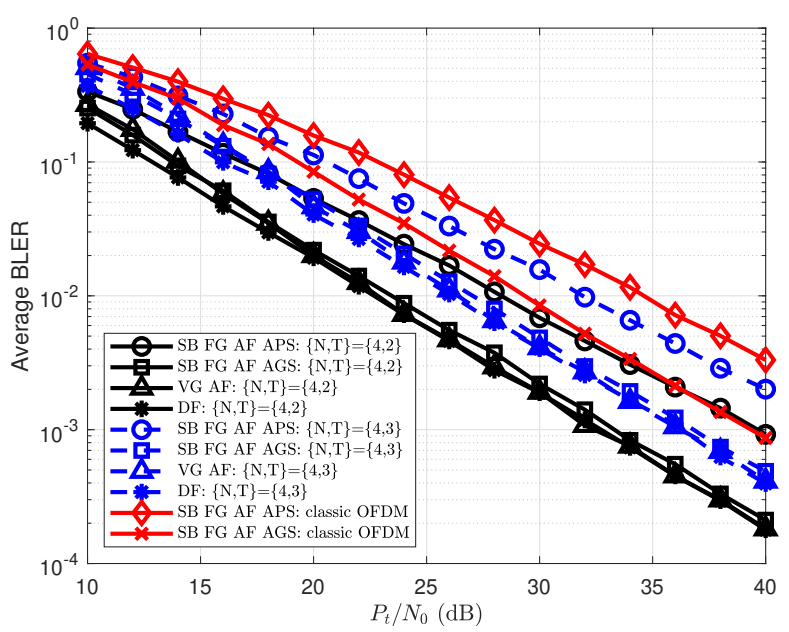

(b) QPSK

Fig. 5: Comparison of average BLER among different multi-carrier relay systems. 


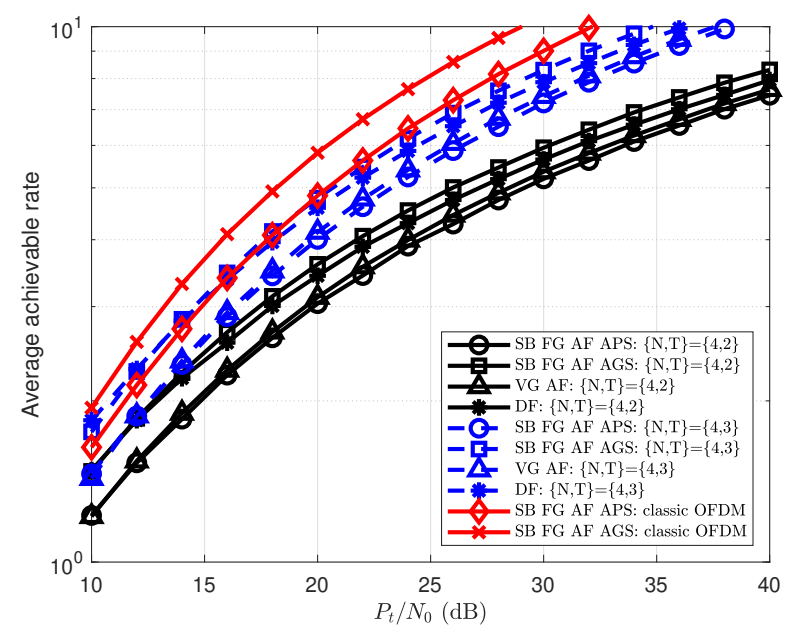

Fig. 6: Comparison of average achievable rate among different multicarrier relay systems.

this paper, which does not need to perform complex decoding and channel estimation at the relay node, but only requires a pre-processing capability at the relay node. In this way, the system complexity can be reduced and the forwarding delay as well as power consumption caused by processing at the relay node also decline. We analyzed the average outage probability, BLER, and achievable rate of the proposed system, and all analytical results are presented in closed form. We also carried out numerical simulations to substantiate the analytical results and compare the FG AF relay assisted OFDM-IM with a series of performance benchmarks. The analysis and numerical results presented in this paper showed a promising vision of FG AF relay assisted OFDM-IM for some new network paradigms, e.g., the IoT, MTC networks and WSNs, where nodes are simple and could not perform complicated processing because of the constraints on power supply and/or hardware configurations.

Although we mentioned the complexity-performance tradeoff by involving $\mathrm{AF}$ and $\mathrm{DF}$ relaying in this paper, only qualitative elaboration was given. More in-depth and quantitative investigations are on demand and worthwhile in order to fully testify the constructive effects brought by FG AF relaying on OFDM-IM. Meanwhile, the optimization of the fixed amplification gain is also worth investigating as future work, since there exists a trade-off between energy consumption and system performance. In addition, advanced application scenarios of FG AF relying are of interest and could be explored to further enhance the performance of FG AF relay assisted OFDM-IM, e.g., multi-user and multi-relay networks.

\section{APPENDIX A}

\section{DERIVATION OF THE CLOSED-FORM EXPRESSION OF $V(a, b, z)$ FOR Two SPECIAL CASES}

To derive the closed-form expression of $V(a, b, z)=$ $\frac{\partial U(a, b, z)}{\partial a}$, we first express $U(a, b, z)$ in an alternative way as

$$
U(a, b, z)=\frac{\Omega(a, b, z)}{\Gamma(a)},
$$

where

$$
\Omega(a, b, z)=\int_{0}^{\infty} e^{-z t} t^{a-1}(1+t)^{b-a-1} \mathrm{~d} t=\Gamma(a) U(a, b, z)
$$

is another defined function that is a closed-form expression and $\Gamma(a)=\int_{0}^{\infty} t^{a-1} e^{-t} \mathrm{~d} t$ is the gamma function. Therefore, $V(a, b, z)$ can be rewritten as

$$
\begin{aligned}
V(a, b, z) & =\frac{\partial U(a, b, z)}{\partial a}=\frac{\partial}{\partial a}\left(\frac{\Omega(a, b, z)}{\Gamma(a)}\right) \\
& =\frac{\frac{\partial \Omega(a, b, z)}{\partial a} \Gamma(a)-\Omega(a, b, z) \frac{\partial \Gamma(a)}{\partial a}}{\Gamma(a)^{2}}
\end{aligned}
$$

where

$$
\frac{\partial \Gamma(a)}{\partial a}=\Gamma(a) \psi_{0}(a)
$$

and $\psi_{n}(\cdot)$ denotes the $n$ th-order polygamma function.

For $\frac{\partial \Omega(a, b, z)}{\partial a}$, we resort to the Leibniz integral rule to derive the closed form expression. Specifically, we have the interchangeable relation between differentiation and integration for $\Omega(a, b, z)$ as follows:

$$
\begin{aligned}
\frac{\partial \Omega(a, b, z)}{\partial a} & =\frac{\partial}{\partial a}\left(\int_{0}^{\infty} e^{-z t} t^{a-1}(1+t)^{b-a-1} \mathrm{~d} t\right) \\
& =\int_{0}^{\infty} \frac{\partial}{\partial a}\left(e^{-z t} t^{a-1}(1+t)^{b-a-1}\right) \mathrm{d} t \\
& =\int_{0}^{\infty} \frac{t^{a-1} \exp (-z t)}{(t+1)^{a+1-b}} \log \left(\frac{t}{t+1}\right) \mathrm{d} t .
\end{aligned}
$$

Unfortunately, to the best of authors' knowledge, there is no a general solution of the integral in (37), $\forall a, b \in \mathbb{Z}$. When $\{a, b\}=\{1,0\}$ and $z>0$, we can derive the closed form expression of $\left.\frac{\partial \Omega(a, 0, z)}{\partial a}\right|_{a=1}$ as follows:

$$
\begin{aligned}
& \left.\frac{\partial \Omega(a, 0, z)}{\partial a}\right|_{a=1}=\int_{0}^{\infty} \frac{\exp (-z t)}{(t+1)^{2}} \log \left(\frac{t}{t+1}\right) \mathrm{d} t \\
& =\exp (z)\left[\begin{array}{l|l}
-G_{2,3}^{3,0}(z & \left.\begin{array}{c}
2,2 \\
0,1,1
\end{array}\right)
\end{array}\right. \\
& \quad+G_{2,3}^{3,0}\left(\begin{array}{c|c}
z & \left.\begin{array}{c}
2,2 \\
1,1,1
\end{array}\right)+(\gamma z+z \log (z)-1) \Gamma(0, z)
\end{array}\right] \\
& -\log (z)-\gamma,
\end{aligned}
$$

where $G_{p, q}^{m, n}\left(z \mid \begin{array}{c}a_{1}, \ldots, a_{p} \\ b_{1}, \ldots, b_{q}\end{array}\right)$ is the Meijer G-function.

Similarly, When $\{a, b\}=\{1,1\}$ and $z>0$, we can derive the closed form expression of $\left.\frac{\partial \Omega(a, 1, z)}{\partial a}\right|_{a=1}$ as

$$
\begin{aligned}
& \left.\frac{\partial \Omega(a, 1, z)}{\partial a}\right|_{a=1}=\int_{0}^{\infty} \frac{\exp (-z t)}{t+1} \log \left(\frac{t}{t+1}\right) \mathrm{d} t \\
& =-\exp (z)\left(\begin{array}{l|c}
\left.2 G_{2,3}^{3,0}\left(\begin{array}{c}
1,1 \\
0,0,0
\end{array}\right)+(\log (z)+\gamma) \Gamma(0, z)\right) .
\end{array}\right.
\end{aligned}
$$

Finally, when $z>0$, substituting (34), (36) as well as (38) or (39) into (35) yields the closed-form expression of $V(1,0, z)$ or $V(1,1, z)$, respectively. 


\section{ACKNOWLEDGMENT}

The authors thank the editor and the anonymous reviewers for their constructive comments that help improve the quality of this manuscript.

\section{REFERENCES}

[1] A. A. Zaidi, R. Baldemair, H. Tullberg, H. Bjorkegren, L. Sundstrom, J. Medbo, C. Kilinc, and I. D. Silva, "Waveform and numerology to support 5G services and requirements," IEEE Communications Magazine, vol. 54, no. 11, pp. 90-98, Nov. 2016.

[2] J. G. Andrews, S. Buzzi, W. Choi, S. V. Hanly, A. Lozano, A. C. K Soong, and J. C. Zhang, "What will 5G be?" IEEE Journal on Selected Areas in Communications, vol. 32, no. 6, pp. 1065-1082, June 2014.

[3] X. Cheng, M. Zhang, M. Wen, and L. Yang, "Index modulation for 5G: striving to do more with less," IEEE Wireless Communications, vol. 25 no. 2, pp. 126-132, Apr. 2018.

[4] R. Y. Mesleh, H. Haas, S. Sinanovic, C. W. Ahn, and S. Yun, "Spatial modulation," IEEE Transactions on Vehicular Technology, vol. 57, no. 4, pp. 2228-2241, July 2008.

[5] E. Basar, U. Aygolu, E. Panayırc1, and H. V. Poor, "Orthogonal frequency division multiplexing with index modulation," IEEE Transactions on Signal Processing, vol. 61, no. 22, pp. 5536-5549, Nov. 2013.

[6] E. Basar, "Index modulation techniques for $5 \mathrm{G}$ wireless networks," IEEE Communications Magazine, vol. 54, no. 7, pp. 168-175, July 2016.

[7] N. Ishikawa, S. Sugiura, and L. Hanzo, "Subcarrier-index modulation aided OFDM - will it work?" IEEE Access, vol. 4, pp. 2580-2593, May 2016.

[8] S. Gokceli, E. Basar, M. Wen, and G. K. Kurt, "Practical implementation of index modulation-based waveforms," IEEE Access, vol. 5, pp. 25463 25 473, Nov. 2017.

[9] M. Wen, X. Cheng, M. Ma, B. Jiao, and H. V. Poor, "On the achievable rate of OFDM with index modulation," IEEE Transactions on Signal Processing, vol. 64, no. 8, pp. 1919-1932, Apr. 2016.

[10] R. Fan, Y. J. Yu, and Y. L. Guan, "Generalization of orthogonal frequency division multiplexing with index modulation," IEEE Transactions on Wireless Communications, vol. 14, no. 10, pp. 5350-5359, Oct. 2015.

[11] M. Wen, Y. Zhang, J. Li, E. Basar, and F. Chen, "Equiprobable subcarrier activation method for OFDM with index modulation," IEEE Communications Letters, vol. 20, no. 12, pp. 2386-2389, Dec. 2016.

[12] M. Wen, B. Ye, E. Basar, Q. Li, and F. Ji, "Enhanced orthogonal frequency division multiplexing with index modulation," IEEE Transactions on Wireless Communications, vol. 16, no. 7, pp. 4786-4801, July 2017

[13] E. Basar, "OFDM with index modulation using coordinate interleaving," IEEE Wireless Communications Letters, vol. 4, no. 4, pp. 381-384, Aug. 2015.

[14] M. Wen, E. Basar, Q. Li, B. Zheng, and M. Zhang, "Multiple-mode orthogonal frequency division multiplexing with index modulation," IEEE Transactions on Communications, vol. 65, no. 9, pp. 3892-3906, Sept. 2017.

[15] Q. Ma, Y. Xiao, L. Dan, P. Yang, L. Peng, and S. Li, "Subcarrier allocation for OFDM with index modulation," IEEE Communications Letters, vol. 20, no. 7, pp. 1469-1472, July 2016.

[16] S. Dang, G. Chen, and J. P. Coon, "Lexicographic codebook design for OFDM with index modulation," IEEE Transactions on Wireless Communications, 2018.

[17] E. Basar, M. Wen, R. Mesleh, M. D. Renzo, Y. Xiao, and H. Haas, "Index modulation techniques for next-generation wireless networks," IEEE Access, vol. 5, pp. 16693-16746, Aug. 2017.

[18] J. Mrkic, E. Kocan, and M. Pejanovic-Djurisic, "Index modulation techniques in OFDM relay systems for 5G wireless networks," in Proc. IEEE TSP, Barcelona, Spain, July 2017, pp. 208-211.

[19] Q. Ma, P. Yang, L. Dan, X. He, Y. Xiao, and S. Li, "OFDM-IM-aided cooperative relaying protocol for cognitive radio networks," in Proc. IEEE SPAWC, Sapporo, Japan, 2017, pp. 1-5.

[20] S. Dang, J. P. Coon, and G. Chen, "Adaptive OFDM with index modulation for two-hop relay-assisted networks," IEEE Transactions on Wireless Communications, vol. 17, no. 3, pp. 1923-1936, Mar. 2018.

[21] S. Dang, G. Chen, and J. P. Coon, "Power allocation for adaptive OFDM index modulation in cooperative networks," in Proc. IEEE GLOBECOM, Singapore, Dec. 2017, pp. 1-6.
[22] J. Crawford and Y. Ko, "Cooperative OFDM-IM relay networks with partial relay selection under imperfect CSI," IEEE Transactions on Vehicular Technology, vol. 67, no. 10, pp. 9362-9369, Oct. 2018.

[23] S. Dang, G. Chen, and J. P. Coon, "Outage performance of two-hop OFDM with index modulation and multi-carrier relay selections," IEEE Wireless Communications Letters, 2018, to appear.

[24] Z. Wang, S. Dang, and D. T. Kennedy, "Multi-hop index modulationaided OFDM with decode-and-forward relaying," IEEE Access, vol. 6, pp. 26457-26468, 2018.

[25] J. Zhao, S. Dang, and Z. Wang, "Full-duplex relay-assisted orthogonal frequency-division multiplexing with index modulation," IEEE Systems Journal, pp. 1-12, 2018.

[26] M. Pejanovic-Djurisic, E. Kocan, and R. Prasad, OFDM Based Relay Systems for Future Wireless Communications. River Publishers, 2012.

[27] Y. Yang, D. Fei, and S. Dang, "Inter-vehicle cooperation channel estimation for IEEE 802.11p V2I communications," Journal of Communications and Networks, vol. 19, no. 3, pp. 227-238, June 2017.

[28] J. Huang, C. Xing, S. Y. Shin, F. Hou, and C. Hsu, "Optimizing M2M communications and quality of services in the IoT for sustainable smart cities," IEEE Transactions on Sustainable Computing, vol. 3, no. 1, pp. 4-15, Jan. 2018.

[29] J. Liu, W. Zhou, and L. Song, "A novel congestion reduction scheme for massive machine-to-machine communication," IEEE Access, vol. 5, pp. 18765-18777, 2017.

[30] M. Elsaadany, A. Ali, and W. Hamouda, "Cellular LTE-A technologies for the future internet-of-things: physical layer features and challenges,' IEEE Communications Surveys \& Tutorials, vol. 19, no. 4, pp. 2544 2572, Fourth quarter 2017.

[31] G. Chen, P. Xiao, J. R. Kelly, B. Li, and R. Tafazolli, "Full-duplex wireless-powered relay in two way cooperative networks," IEEE Access, vol. 5, pp. 1548-1558, 2017.

[32] M. O. Hasna and M. . Alouini, "A performance study of dual-hop transmissions with fixed gain relays," IEEE Transactions on Wireless Communications, vol. 3, no. 6, pp. 1963-1968, Nov. 2004.

[33] G. Farhadi and N. C. Beaulieu, "On the performance of amplify-andforward cooperative systems with fixed gain relays," IEEE Transactions on Wireless Communications, vol. 7, no. 5, pp. 1851-1856, May 2008.

[34] M. D. Renzo, F. Graziosi, and F. Santucci, "A comprehensive framework for performance analysis of dual-hop cooperative wireless systems with fixed-gain relays over generalized fading channels," IEEE Transactions on Wireless Communications, vol. 8, no. 10, pp. 5060-5074, Oct. 2009.

[35] H. A. Suraweera, D. S. Michalopoulos, and G. K. Karagiannidis, "Performance of distributed diversity systems with a single amplifyand-forward relay," IEEE Transactions on Vehicular Technology, vol. 58, no. 5, pp. 2603-2608, June 2009.

[36] P. L. Yeoh, M. Elkashlan, and I. B. Collings, "Selection relaying with transmit beamforming: A comparison of fixed and variable gain relaying," IEEE Transactions on Communications, vol. 59, no. 6, pp. 1720-1730, June 2011.

[37] J. P. Coon, "A theorem on the asymptotic outage behavior of fixed-gain amplify-and-forward relay systems," IEEE Communications Letters, vol. 18, no. 9, pp. 1567-1570, Sept. 2014.

[38] S. Berger, M. Kuhn, A. Wittneben, T. Unger, and A. Klein, "Recent advances in amplify-and-forward two-hop relaying," IEEE Communications Magazine, vol. 47, no. 7, pp. 50-56, July 2009.

[39] M. Elkashlan, P. L. Yeoh, R. H. Y. Louie, and I. B. Collings, "On the exact and asymptotic ser of receive diversity with multiple amplify-andforward relays," IEEE Transactions on Vehicular Technology, vol. 59, no. 9, pp. 4602-4608, Nov. 2010.

[40] A. Koc, I. Altunbas, and E. Basar, "Two-way full-duplex spatial modulation systems with wireless powered AF relaying," IEEE Wireless Communications Letters, vol. 7, no. 3, pp. 444-447, June 2018.

[41] A. Chandra, C. Bose, and M. K. Bose, "Wireless relays for next generation broadband networks," IEEE Potentials, vol. 30, no. 2, pp. 39-43, Mar. 2011.

[42] G. Huang, Y. Wang, and J. Coon, "Performance of multihop decodeand-forward and amplify-and-forward relay networks with channel estimation," in Proc. IEEE PacRim, Aug. 2011, pp. 352-357.

[43] P. Yang, M. D. Renzo, Y. Xiao, S. Li, and L. Hanzo, "Design guidelines for spatial modulation," IEEE Communications Surveys \& Tutorials, vol. 17, no. 1, pp. 6-26, First quarter 2015.

[44] M. D. Renzo and H. Haas, "Bit error probability of SM-MIMO over generalized fading channels," IEEE Transactions on Vehicular Technology, vol. 61, no. 3, pp. 1124-1144, Mar. 2012.

[45] B. Zhou, H. Hu, and H. Wang, "Cyclic prefix update for OFDM amplifyand-forward relay systems," Wireless personal communications, vol. 72 , no. 4, pp. 2281-2294, 2013. 
[46] Y. Ding and M. Uysal, "Amplify-and-forward cooperative OFDM with multiple-relays: performance analysis and relay selection methods," IEEE Transactions on Wireless Communications, vol. 8, no. 10, pp. 4963-4968, Oct. 2009.

[47] G. Wang, F. Gao, Y. Wu, and C. Tellambura, "Joint CFO and channel estimation for OFDM-based two-way relay networks," IEEE Transactions on Wireless Communications, vol. 10, no. 2, pp. 456-465, Feb. 2011.

[48] A. Chowdhery and R. K. Mallik, "Linear detection for the nonorthogonal amplify and forward protocol," IEEE Transactions on Wireless Communications, vol. 8, no. 2, pp. 826-835, Feb. 2009.

[49] W. Yang and Y. Cai, "On the performance of the block-based selective OFDM decode-and-forward relaying scheme for $4 \mathrm{G}$ mobile communication systems," Journal of Communications and Networks, vol. 13, no. 1, pp. 56-62, Feb. 2011

[50] W. Wang and R. Wu, "Capacity maximization for OFDM two-hop relay system with separate power constraints," IEEE Transactions on Vehicular Technology, vol. 58, no. 9, pp. 4943-4954, Nov. 2009.

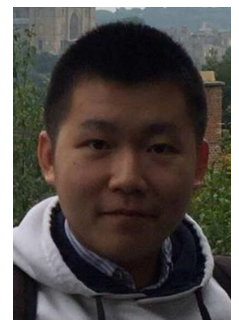

Shuping Dang (S'13-M'18) received B.Eng (Hons) in Electrical and Electronic Engineering from the University of Manchester (with first class honors) and B.Eng in Electrical Engineering and Automation from Beijing Jiaotong University in 2014 via a joint ' $2+2$ ' dual-degree program. He also received D.Phil in Engineering Science from University of Oxford in 2018. Dr. Dang joined in the R\&D Center, Huanan Communication Co., Ltd. after graduating from University of Oxford and is currently working as a Postdoctoral Fellow with the Computer, Electrical and Mathematical Science and Engineering Division, King Abdullah University of Science and Technology (KAUST). He serves as a reviewer for a number of key journals in communications and information science, including IEEE TRANSACTIONS ON WIRELESS COMMUNICATIONS, IEEE TRANSACTIONS ON COMMUNICATIONS and IEEE TRANSACTIONS ON VEHICULAR TECHNOLOGY. His current research interests include artificial intelligence assisted communications, novel modulation schemes and cooperative communications.

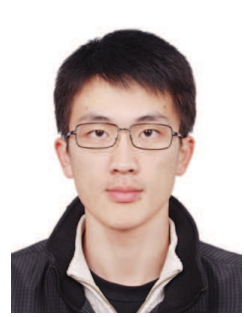

Miaowen Wen (M'14-SM'18) received the B.S. degree from Beijing Jiaotong University, Beijing, China, in 2009, and the Ph.D. degree from Peking University, Beijing, China, in 2014. From 2012 to 2013, he was a Visiting Student Research Collaborator with Princeton University, Princeton, NJ, USA. $\mathrm{He}$ is currently an Associate Professor with the South China University of Technology, Guangzhou, China. He has authored a book and more than 60 journal papers. His research interests include index modulation, non-orthogonal multiple access, physical layer security, and molecular communications.

Dr. Wen was the recipient of the Excellent Doctoral Dissertation Award from Peking University and the Best Paper Awards from the IEEE ITST'2012, the IEEE ITSC'2014, and the IEEE ICNC'2016. He was recognized as an Exemplary Reviewer for the IEEE Communications Letters in 2017. He served as a symposium co-chair for the IEEE ICNC'2019, a workshop cochair for the IEEE/CIC ICCC'2018, and a Guest Editor for IEEE Journal on Selected Areas in Communications (Special Issue on Spatial Modulation for Emerging Wireless Systems) and for IEEE Journal of Selected Topics in Signal Processing (Special Issue on Index Modulation for Future Wireless Networks: A Signal Processing Perspective). He has served on the Editorial Boards of several international journals, including the IEEE Access, the EURASIP Journal on Wireless Communications and Networking, the ETRI Journal, and the Physical Communication (Elsevier).

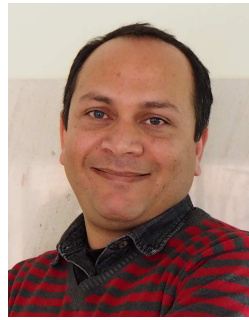

Shahid Mumtaz (SM'16) has more than ten years of wireless industry experience and is currently working as Senior Research Scientist at Instituto de Telecomunicações, Aveiro, Portugal. Prior to his current position, he worked as Research Intern at Ericsson and Huawei Research Labs in 2005 at Karlskrona, Sweden. He received his MSc and Ph.D. degrees in Electrical \& Electronic Engineering from Blekinge Institute of Technology (BTH) Karlskrona, Sweden and University of Aveiro, Portugal in 2006 and 2011, respectively. Dr. Mumtaz has more than 150 publications in international conferences, journal papers, and book chapters. Dr. Mumtaz is a senior member of IEEE. He was awarded an 'Alain Bensoussan' fellowship by ERCIM to pursue research in communication networks for one year at the VTT Technical Research Centre of Finland in 2012. He was nominated as Vice Chair for IEEE new standardization on P1932.1: Standard for Licensed/Unlicensed Spectrum Interoperability in Wireless Mobile Networks. He is also actively involved in 3GPP standardization on LTE release 12 onwards, along with major manufacturers. He is an ACM distinguished speaker.

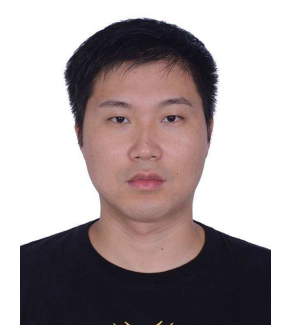

Jun Li (S'13-M'17) received the M.S. and the Ph.D. degrees from Chonbuk National University, Jeonju, South Korea, in 2011 and 2017, respectively. He has been an Assistant Professor with Guangzhou University, Guangzhou, China. He had participated as the Vice Head Researcher in the World Class University Project, sponsored by the National Research Foundation of Korea Grant funded by the Korean Ministry of Education Science and Technology. His research interests include spatial modulation and OFDM index modulation.

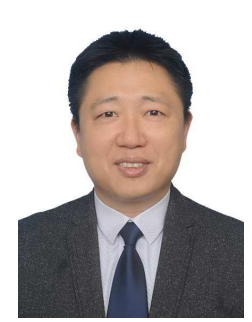

Zhenrong Zhang (M'18) received his B.S. and M.S. from Electronics Department, Peking University, China, in 1998 and 2001, respectively, and the Ph.D degree from Nanyang Technological University (NTU), Singapore in 2006. Currently, he is a Postdoctoral Research Associate in the National Laboratory on Local Optic-fiber Communication Networks and Advanced Optical Communication Systems, Department of Electronics, Peking University and also a professor with School of Computer, Electronic and Information, Guangxi University (GXU). He has published over 20 technical papers in journals and conferences. His research interests include survivability and restoration in optical networks, network design, protocol development for Optical Networks and wireless communications. 